\title{
Originales
}

\section{Factor de von Willebrand, lesión endotelial y cardiopatía isquémica}

\author{
B. VIRGÓS-SEÑOR ${ }^{a}$, A. NEBRA-PUERTAS ${ }^{a}$, M.A. SUÁREZ-PINILLA ${ }^{b}$, R. CORNUDELLA-LACASA ${ }^{c}$ \\ Y P. PORTERO-PÉREZ ${ }^{d}$
}

aServicio de Medicina Intensiva. Hospital Universitario Miguel Servet. Zaragoza. España. Servicios de bedicina Intensiva, cHematología y dCardiología. Hospital Clínico Universitario. Zaragoza. España.

Objetivo. Analizar si los niveles de factor de von Willebrand (FvW) son más elevados en pacientes con cardiopatía isquémica que en individuos sanos, y evaluar la relación de dichos niveles con la recidiva clínica de angina y el intervencionismo coronario.

Diseño. Estudio prospectivo observacional.

Pacientes. Analizamos los niveles de FvW en 75 pacientes con cardiopatía isquémica sometidos a intervencionismo coronario (Grupo I), y los comparamos con los de 30 individuos sanos, sin factores de riesgo cardiovascular, y libres teóricamente de lesiones coronarias (Grupo II).

Variables principales. Niveles de FvW antes de la realización del intervencionismo coronario (toma 0), a las 24 horas (toma 1) y a los tres meses durante el seguimiento ambulatorio (toma 2). En el Grupo II se efectuó una única determinación de los niveles de FvW.

Resultados. Los sujetos con cardiopatía isquémica tuvieron unos niveles más elevados de FvW que los sujetos sanos (162 $\pm 74 \%$ frente a $95 \pm$ $33 \% ; p=0,0001)$. Los niveles de FvW aumentaron de manera significativa tras el intervencionismo coronario $(162,4 \pm 74,9 \%$ en la toma 0 frente a 213 $\pm 90 \%$ en la toma $1 ; p=0,0001$ ). Los pacientes sintomáticos a los tres meses no presentaron diferencias significativas respecto a los asintomáticos en los niveles de FvW (125 $\pm 63 \%$ frente a 133 $\pm 60 \% ; p=0,57$ ).

Conclusiones. Los niveles de FvW reflejan una alteración endotelial en pacientes con cardiopatía

\footnotetext{
Correspondencia: Dra. B. Virgós Señor. Avda. de Valencia, 51-53. Esc. 1. ${ }^{\mathrm{a}}, 8{ }^{\circ} \mathrm{B}$. 50005 Zaragoza. España.

Correo electrónico: beaagus@wanadoo.es

Manuscrito aceptado el 15-X-2007.
}

isquémica. El aumento de los niveles tras el intervencionismo coronario podría ser debido a la propia agresión endotelial del intervencionismo. No se demuestran mayores niveles de FvW en pacientes sintomáticos en el seguimiento a tres meses.

PALABRAS CLAVE: cardiopatía isquémica, lesión endotelial, factor de von Willebrand.

\section{VON WILLEBRAND FACTOR, ENDOTHELIAL LESION, AND ISCHEMIC HEART DISEASE}

Objective. To analyze if the levels of von Willebrand factor (FvW) are higher in patients with ischemic heart disease than in healthy subjects and evaluate the relationship of these levels with clinical recurrence and coronary interventionism.

Design. Observational prospective study.

Patients. We analyzed the levels of FvW in 75 patients with ischemic heart disease who underwent coronary interventionism (Group I) and compared them with those of 30 healthy subjects with no cardiovascular risk factors and who, theoretically, had no coronary injuries (Group II).

Main variables. Levels of FvW before coronary interventionism (sample 0), 24 hours after (sample 1), and at three months of out-patient follow-up (sample 2). A single measurement was made of the FvW levels in Group II.

Results. Subjects with ischemic heart disease had higher levels of FvW than healthy subjects $(162 \pm 74 \%$ versus $95 \pm 33 \% ; p=0.0001)$. FvW levels were significantly increased after coronary interventionism $(162.4 \pm 74.9 \%$ in sample 0 versus $213 \pm 90 \%$ in sample $1 ; p=0.0001$ ). Patients with clinical symptoms at three months have no significant difference regarding those with no symptoms in the FvW levels (125 $\pm 63 \%$ versus $133 \pm$ $60 \% ; p=0.57$ ). 
Conclusions. FvW levels reflect an endothelial alteration in patients with ischemic heart disease. The increase of the levels after coronary interventionism could be due to the endothelial aggression itself of the intervention. It was not possible to demonstrate higher levels of FvW in patients with symptoms in the three month follow-up.

KEY WORDS: ischemic heart disease, endothelial injury, von Willebrand factor.

\section{INTRODUCCIÓN}

La lesión endotelial es el substrato fisiopatológico de la inmensa mayoría de los pacientes con cardiopatía isquémica $(\mathrm{CI})^{1-3}$. El factor de von Willebrand $(\mathrm{FvW})$ es una molécula proteica localizada a nivel subendotelial, con diversas funciones dentro de la hemostasia sanguínea, y cuya elevación puede ser observada en situaciones en las que se produce una lesión endotelial ${ }^{4-12}$. En relación con la fisiopatología de la CI, en la que se produce una alteración endotelial, algunos estudios demuestran la existencia de una elevación en los niveles de FvW en estos pacientes, aunque con resultados diferentes en función del momento evolutivo y de la presentación clínica, existiendo también variaciones en cuanto al tratamiento recibido por los enfermos en los distintos trabajos ${ }^{13-16}$.

Entre las herramientas diagnósticas y terapéuticas para pacientes con CI, disponemos de la coronariografía, acompañada en un porcentaje elevado de casos de técnicas de intervencionismo coronario.

Nosotros hemos analizado los niveles de FvW en sujetos con CI y en sujetos sanos para investigar si dichos niveles son superiores en los pacientes con CI, y si los niveles se relacionan con la persistencia o no de la clínica anginosa, a los tres meses del intervencionismo coronario.

\section{PACIENTES Y MÉTODOS}

Estudio observacional realizado con 75 pacientes diagnosticados de CI sometidos a intervencionismo coronario (Grupo I) y un grupo control de 30 sujetos sanos, sin factores de riesgo cardiovascular conocidos ni, teóricamente, lesiones coronarias (Grupo II). El diagnóstico de CI se realizó por criterios clínicos y/o electrocardiográficos compatibles con angor, y se utilizó la clasificación de Braunwald ${ }^{17}$ para catalogar la situación clínica que justificó la realización del cateterismo (tabla 1). En $42(56 \%)$ pacientes el cateterismo fue solicitado de manera ambulatoria. El intervencionismo coronario percutáneo realizado fue angioplastia (ACTP) sola en $21(28 \%)$ casos y angioplastia con stenting en los $54(72 \%)$ casos restantes. En $16(21 \%)$ casos se hizo, además, aterotomía. En $14(19 \%)$ sujetos se utilizaron los inhibidores de las glucoproteínas IIb-IIIa. Se analizó la relación de los niveles de FvW con el número de lesiones coronarias significativas halladas en el cateterismo, considerándose como significativas aquellas lesiones con una ocupación angiográfica de al menos el $60 \%$ de la luz del vaso.

Los sujetos del Grupo II fueron seleccionados de manera aleatoria entre personas sanas que acudieron como acompañantes a las consultas externas de nuestro hospital. A estos sujetos se les realizó una pequeña anamnesis, una breve exploración física que incluía la medición de la presión arterial y un estudio analítico para descartar alteraciones lipídicas y/o hiperglucemia.

Las venopunciones para obtener las muestras sanguíneas para determinación del FvW se efectuaron de manera atraumática, centrifugándose a $3.600 \mathrm{rpm}$ durante 10 minutos; el plasma así obtenido se congeló en una seroteca a $-80^{\circ} \mathrm{C}$ hasta su posterior utilización.

El FvW fue cuantificado en plasma mediante técnica automatizada de turbidimetría de partículas de látex (test de IL-Test ${ }^{\circledR}$, en un autoanalizador ACL Futura $^{\circledR}$ ). Dicha técnica determina la presencia de FvW antigénico, ofreciendo los resultados en porcentaje de actividad del FvW, tras calibrar el sistema con los sueros control.

En el Grupo I analizamos los niveles de FvW de manera previa a la realización del intervencionismo coronario (toma 0 ), a las 24 horas (toma 1 ) y a los tres meses durante el seguimiento ambulatorio (toma 2). En el Grupo II se efectuó una única determinación de los niveles de FvW.

Además de cuantificar los niveles de FvW, se realizó de manera basal a todos los sujetos del estudio un hemograma completo, un análisis de coagulación estándar (fibrinógeno, tiempo de tromboplastina parcial activada, porcentaje de actividad de protrombina) y una determinación de niveles de glucosa, urea, creatinina, iones, niveles de colesterol ligado a lipoproteínas de alta densidad (c-HDL) y colesterol total.

El antecedente de CI fue clasificado en tres grupos: SCASEST, angor estable e infarto agudo de

TABLA 1. Clasificación Braunwald de angina inestable

\begin{tabular}{|l|c|c|c|}
\hline & $\begin{array}{c}\text { Angina secundaria o con causa } \\
\text { extracoronaria desencadenante }\end{array}$ & $\begin{array}{c}\text { Angina primaria o sin causa } \\
\text { extracoronaria desencadenante }\end{array}$ & $\begin{array}{c}\text { Angina posinfarto } \\
\text { (IAM en las dos semanas previas) }\end{array}$ \\
\hline $\begin{array}{l}\text { Angina reciente severa, pero no de reposo } \\
\text { Angina de reposo en el último mes, pero no }\end{array}$ & IA & IB & IC \\
en las últimas 48 horas & IIA & IIB & IIC \\
Angina de reposo en las últimas 48 horas & IIIA & IIIB & IIIC \\
\hline
\end{tabular}

Cada categoría de la tabla se complementa según el tratamiento: 1: tratamiento insuficiente; 2: tratamiento estándar correcto; 3: tratamiento máximo-triple terapia. IAM: infarto agudo de miocardio. 
TABLA 2. Clasificación funcional de la Canadian Cardiovascular Society

$\begin{array}{|ll|}\text { Grado I } & \text { La actividad física normal no causa dolor; este aparece con los esfuerzos extenuantes, rápidos o prolongados } \\ \text { Grado II } & \begin{array}{l}\text { Limitación leve de la actividad física; el dolor aparece al caminar con paso normal dos o más travesías o subir más de un } \\ \text { piso }\end{array} \\ \text { Grado III } & \begin{array}{l}\text { Limitación importante de la capacidad funcional; el dolor se presenta al subir un piso o caminar con paso normal una } \\ \text { travesía }\end{array} \\ \text { Grado IV } & \text { Incapacidad para llevar a cabo cualquier actividad física sin la aparición de angina; el dolor puede aparecer en reposo }\end{array}$

TABLA 3. Características de los pacientes incluidos en el estudio

\begin{tabular}{|c|c|c|}
\hline & Grupo I $(n=75)$ & Grupo II $(\mathrm{n}=30)$ \\
\hline Edad, años (media \pm DE) & $60,6 \pm 10,3$ & $58,1 \pm 11,5$ \\
\hline Varones & $68(91 \%)$ & $28(93 \%)$ \\
\hline \multicolumn{3}{|l|}{ Factores de riesgo cardiovascular } \\
\hline Dislipidemia & $38(51 \%)$ & \\
\hline Hipertensión arterial & $25(33 \%)$ & \\
\hline Diabetes mellitus & $22(29 \%)$ & \\
\hline Tabaquismo & $44(59 \%)$ & \\
\hline Enolismo & $5(10 \%)$ & \\
\hline Varios factores & $63(84 \%)$ & \\
\hline \multicolumn{3}{|l|}{$\begin{array}{l}\text { Antecedentes de cardiopatía } \\
\text { isquémica }\end{array}$} \\
\hline SCASEST & $4(5 \%)$ & \\
\hline Angina estable & $18(24 \%)$ & \\
\hline IAM transmural & $39(52 \%)$ & \\
\hline \multicolumn{3}{|l|}{ Tratamiento médico } \\
\hline AAS & $75(100 \%)$ & \\
\hline Dihidropiridinas & $11(15 \%)$ & \\
\hline Fibrinolisis 10 días previos & $17(23 \%)$ & \\
\hline Heparina & $23(31 \%)$ & \\
\hline Calcioantagonistas & $45(60 \%)$ & \\
\hline Bloqueadores beta & $23(31 \%)$ & \\
\hline Nitritos & $65(87 \%)$ & \\
\hline Hipolipidemiantes & $38(51 \%)$ & \\
\hline IECA & $11(15 \%)$ & \\
\hline \multicolumn{3}{|l|}{ Inhibidores de glucoproteínas } \\
\hline IIb-IIIa & $14(19 \%)$ & \\
\hline
\end{tabular}

AAS: ácido acetilsalicílico; DE: desviación estándar; IAM: infarto agudo de miocardio; IECA: inhibidores de la enzima de conversión de la angiotensina.

miocardio (IAM) transmural. Dentro de los factores de riesgo cardiovascular estudiados, se consideró como dislipidémicos y/o hipertensos a aquellos pacientes en tratamiento médico previo. Para aceptar el tabaquismo como factor de riesgo se exigió o bien que el hábito fuera activo en el momento del estudio, o bien que los pacientes hubieran fumado al menos 10 cigarrillos/día en los 5 años previos. Se definieron como diabéticos aquellos enfermos que en el momento del estudio estuvieran en tratamiento, bien con insulina, bien con antidiabéticos orales. Se aceptó que la toma de alcohol supuso un factor de riesgo cardiovascular en aquellos enfermos con una ingesta diaria de al menos $50 \mathrm{~g} /$ día de etanol.

Se estudió la posible influencia del tratamiento médico seguido por los enfermos, analizando la relación que pudiera existir entre los niveles de FvW y el tratamiento con dihidropiridinas, heparina, administración de dextranos, fibrinolisis en los 10 días previos al estudio, calcioantagonistas, bloqueadores beta, nitritos, hipolipidemiantes e inhibidores de la enzima de conversión de la angiotensina (IECA).
En el seguimiento ambulatorio realizado al tercer mes del intervencionismo realizamos una entrevista clínica valorando la recurrencia de clínica anginosa; se utilizó la clasificación funcional de la Canadian Cardiovascular Society ${ }^{18}$ (tabla 2). A los pacientes con sintomatología se les realizó una ergometría convencional.

\section{Análisis estadístico}

Aplicamos la prueba de la t de Student y ANOVA, y la prueba de Wilcoxon, para datos no ajustados a la normalidad. Se empleó el test de Mood (prueba no paramétrica que compara las medianas de muestras entre más de tres grupos) para comparar determinados resultados intergrupo. Se realizó un análisis multivariable mediante regresión logística tomando como variable dependiente la recurrencia clínica a los tres meses, y como independientes los niveles de FvW en las tres tomas, los resultados del estudio de coagulación, los niveles de colesterol total, los distintos factores de riesgo cardiovascular y el tratamiento médico seguido por los enfermos. Se consideró un valor de p inferior a 0,05 como significativo.

\section{RESULTADOS}

Las características generales de los sujetos de ambos grupos, así como los antecedentes y el tratamiento de los pacientes del Grupo I, se presentan en la tabla 3 .

Los sujetos con CI presentaron unos niveles significativamente superiores de tiempo de tromboplastina parcial activada, fibrinógeno, c-HDL y colesterol total que los sujetos sanos (tabla 4). Además, los pacientes del Grupo I experimentaron un aumento significativo del fibrinógeno en las siguientes 24 horas tras el intervencionismo coronario.

Los sujetos del Grupo I presentaron unos niveles significativamente más elevados de $\mathrm{FvW}$ respecto de los del grupo II (162 $\pm 75 \%$ frente a $96 \pm 33 \%$; p < 0,0001) (tabla 5). En los pacientes del Grupo I, los niveles de FvW aumentaron de manera significativa a las 24 horas del intervencionismo coronario (162 \pm $75 \%$ frente a $213 \pm 90 \%$; p < 0,0001). El descenso en los niveles de $\mathrm{FvW}$ al tercer mes respecto al basal fue significativo $(162 \pm 75 \%$ frente a $128,7 \pm 61,8 \%$; prueba de Mann-Whitney, $\mathrm{p}<0,0001)$.

La influencia de los distintos tratamientos médicos sobre los niveles basales de FvW se puede observar en la tabla 6. Tan solo hubo diferencias en cuanto al porcentaje de actividad de FvW entre los pacientes 
VIRGÓS-SEÑOR B ET AL. FACTOR DE VON WILLEBRAND, LESIÓN ENDOTELIAL Y CARDIOPATÍA ISQUÉMICA

TABLA 4. Resultado de las determinaciones analíticas efectuadas*

\begin{tabular}{|c|c|c|c|}
\hline & Grupo I $(n=75)$ & Grupo II $(\mathrm{n}=30)$ & $\mathrm{p}$ \\
\hline TTPa toma 0 & $41,3 \pm 23,3 \mathrm{seg}$ & $29,8 \pm 2,5 \mathrm{seg}$ & 0,0051 \\
\hline TTPa toma 1 & $52,5 \pm 27,8 \mathrm{seg}$ & - & \\
\hline TTPa toma 2 & $30,8 \pm 5,06 \mathrm{seg}$ & - & \\
\hline Activ. protrb. toma 0 & $89,4 \pm 11,5 \%$ & $97,2 \pm 13,9 \%$ & 0,065 \\
\hline Activ. protrb. toma 1 & $83,6 \pm 12,7 \%$ & - & \\
\hline Activ. protrb. toma 2 & $96,2 \pm 10,5 \%$ & - & \\
\hline Fibrinógeno toma 0 & $413,9 \pm 155 \mathrm{mg} / \mathrm{dl}$ & $313,6 \pm 71,9 \mathrm{mg} / \mathrm{dl}$ & 0,0010 \\
\hline Fibrinógeno toma 1 & $419,5 \pm 133 \mathrm{mg} / \mathrm{dl}$ & - & \\
\hline Fibrinógeno toma 2 & $354,8 \pm 86,4 \mathrm{mg} / \mathrm{dl}$ & - & \\
\hline Plaquetas toma 0 & $196.991 \pm 63.949 / \mathrm{mm}^{3}$ & $270.000 \pm 57.100 / \mathrm{mm}^{3}$ & 0,086 \\
\hline Plaquetas toma 1 & $176.851 \pm 59.838 / \mathrm{mm}^{3}$ & - & \\
\hline Plaquetas toma 2 & $193.973 \pm 55.877 / \mathrm{mm}^{3}$ & - & \\
\hline c-HDL toma 0 & $37,8 \pm 8 \mathrm{mg} / \mathrm{dl}$ & $44,4 \pm 6,9 \mathrm{mg} / \mathrm{dl}$ & 0,002 \\
\hline c-HDL toma 2 & $43,5 \pm 9,3 \mathrm{mg} / \mathrm{dl}$ & - & \\
\hline Colesterol total toma 0 & $200,1 \pm 53,3 \mathrm{mg} / \mathrm{dl}$ & $171,2 \pm 27 \mathrm{mg} / \mathrm{dl}$ & 0,007 \\
\hline Colesterol total toma 2 & $215,4 \pm 42,6 \mathrm{mg} / \mathrm{dl}$ & & \\
\hline
\end{tabular}

*Los valores se presentan como media y desviación estándar. Activ. Protrb: actividad de protrombina; c-HDL: colesterol ligado a lipoproteínas de alta densidad; TTPa: tiempo de tromboplastina parcial activada.

que estaban en tratamiento con heparina en el momento de la extracción de la muestra.

En $44(59 \%)$ pacientes del Grupo I se observó más de una lesión coronaria y en el resto de los pacientes una lesión única. Los niveles basales de FvW fueron significativamente mayores en los pacientes con más de una lesión coronaria que en los pacientes con lesión única $(174 \pm 82 \%$ frente a $145 \pm 12 \%$; p = 0,0002).

Clasificando a los sujetos del Grupo I según las categorías de Braunwald, hubo 17 en el grupo I-B2 $(22,6 \%), 3$ en el I-B3 (4\%), 1 en la categoría II-A2 $(1,3 \%), 21$ en la II-B2 (28\%), 20 en la III-B2 (26,6\%), 2 en la III-B3 (2,6\%), 8 en la III-C2 $(10,6 \%)$ y 3 en la III-C3 (4\%). Los niveles de FvW, según las categorías de Braunwald, fueron de $137 \pm 60 \%$ en el I-B2, $119 \pm 49 \%$ en el II-B2, $202 \pm 74 \%$ en el III-B2, de $201 \pm 82 \%$ en el III-C2, siendo estas diferencias estadísticamente significativas (test de Mood, $\mathrm{p}=$ $0,001)$.

Los niveles de FvW fueron $125 \pm 63 \%$ en los 40 pacientes que se encontraban asintomáticos a los tres meses de la realización del cateterismo frente a $133 \pm$ $60 \%$ en los pacientes que presentaban clínica anginosa $(p=0,57)($ tabla 7$)$. Los niveles de FvW fueron $122 \pm 63 \%$ en los 7 pacientes con ergometría negativa y $131 \pm 67 \%$ en los 28 pacientes con ergometría positiva (prueba de Mann-Whitney, $\mathrm{p}=0,884$ ). En la tabla 7 se muestran los niveles de FvW a los tres meses en los pacientes de acuerdo a su situación funcional según la clasificación de la Canadian Cardiovascular Society.
El análisis multivariante de regresión logística solo mostró asociación significativa entre la recurrencia clínica a los tres meses y la presencia de más de una lesión significativa en la coronariografía (odds ratio 5,92; intervalo de confianza del 95\%: 1,09 a 32,09; p $=0,039$ ).

\section{DISCUSIÓN}

Presentamos un estudio descriptivo que muestra que los pacientes con CI y enfermedad coronaria, comprobada mediante coronariografía, tienen niveles significativamente más elevados de FvW que los sujetos sanos. Estos resultados son coincidentes con los reportados en los trabajos de distintos autores ${ }^{9,10,12-22}$, pudiendo justificarse la elevación del FvW en los pacientes con enfermedad coronaria tanto por la existencia de un estado de hipercoagulabilidad subclínica en este tipo de enfermos, como por la presencia de una lesión endotelial. Tanto el hecho de que se obtengan niveles más elevados de FvW en las categorías de Braunwald más graves, como el que los enfermos con lesiones múltiples tengan mayor actividad de FvW, sugieren que la elevación de los niveles de $\mathrm{FvW}$ es un factor que indica la presencia de lesión endotelial severa y, por lo tanto, mayor gravedad clínica. No obstante, tampoco debemos olvidar que los pacientes de este estudio tenían múltiples factores de riesgo cardiovascular, y que existen trabajos que muestran que la mera existencia de estos factores de riesgo puede promover un incremento en los niveles de $\mathrm{FvW}^{19-22}$.

TABLA 5. Niveles de factor de von Willebrand*

\begin{tabular}{|l|c|c|c|}
\hline & Grupo I $(\mathrm{n}=75)$ & Grupo II $(\mathrm{n}=30)$ & $\mathrm{p}$ \\
\hline FvW en la toma 0 & $162,4 \pm 74 \%$ & $95,83 \% \pm 33,5 \%$ & $\mathrm{p}<0,0001$ \\
FvW en la toma 1 & $212,8 \pm 90,5 \%$ & - & - \\
FvW en la toma 2 & $128,7 \pm 61,8 \%$ & - & - \\
\hline
\end{tabular}

*Los valores se presentan como media y desviación estándar. FvW: factor de von Willebrand. 
TABLA 6. Niveles de factor de von Willebrand en la toma 0 (basal) de acuerdo al tratamiento médico que recibían o no los pacientes estudiados en el Grupo I

\begin{tabular}{|c|c|c|c|}
\hline & FvW en pacientes con tratamiento & FvW en pacientes sin tratamiento & $\mathrm{p}$ \\
\hline Dihidropiridinas & $197,5+92,8$ & $156,4 \pm 70,6$ & 0,19 \\
\hline Fibrinolisis10 días previos & $193,1 \pm 76,5$ & $153,4 \pm 72,7$ & 0,07 \\
\hline Heparina & $214,8 \pm 63,4$ & $143,4 \pm 70,0$ & 0,001 \\
\hline Calcioantagonistas & $164,3 \pm 77,4$ & $159,6 \pm 72,0$ & 0,79 \\
\hline Bloqueadores beta & $143,3 \pm 72,5$ & $170,9 \pm 75,2$ & 0,14 \\
\hline Nitritos & $164,6 \pm 75,7$ & $148,2 \pm 72,5$ & 0,52 \\
\hline Hipolipidemiantes & $159,4 \pm 82,5$ & $162,8 \pm 74,7$ & 0,91 \\
\hline IECA & $192,3 \pm 77,3$ & $157,8 \pm 74,1$ & 0,21 \\
\hline Inhibidores de las glucoproteínas IIb-IIIa* & $213,1 \pm 93,6$ & $211,8 \pm 78,7$ & 0,81 \\
\hline
\end{tabular}

*Niveles de factor de von Willebrand (FvW) en la toma 1. IECA: inhibidores de la enzima de conversión de la angiotensina.

Los pacientes que estaban recibiendo tratamiento con heparina presentaron unos niveles de FvW significativamente superiores a los de los pacientes que no estaban recibiendo dicho tratamiento. Si bien es conocida la interferencia de la heparina en la función normal del FvW impidiendo la unión eficaz de este a sus receptores plaquetarios ${ }^{23,24}$, y que algunos autores consideran que existe una alteración en el FvW antigénico dependiente de la heparina ${ }^{25,26}$, nuestros resultados podrían explicarse por el hecho de que los pacientes que reciben tratamiento con heparina suelen ser los que tienen una lesión endotelial más severa o presentan mayor expresividad clínica. Un resultado contrario al demostrado en nuestra serie, en relación con la influencia del tratamiento heparínico en los niveles de $\mathrm{FvW}$, es el demostrado por Ray et $\mathrm{al}^{27}$, quienes observan un descenso del FvW en pacientes con IAM agudo tratados con enoxaparina. Este resultado no es extrapolable a nuestra serie, puesto que el diseño del trabajo está basado en niveles basales de FvW elevados, durante la fase aguda del IAM.

Nuestro estudio también muestra una elevación significativa de los niveles de fibrinógeno en los pacientes con CI respecto de los pacientes sanos, algo que ya había sido reportado por otros autores en pacientes con enfermedad coronaria ${ }^{28-30}$, habiéndose propuesto esta elevación como marcador de riesgo coronario y como molécula de fase aguda, con lo que también se plantearía la duda de la interpretación del $\mathrm{FvW}$ como un mero reactante de fase aguda. En esta línea argumental, el trabajo de Danesh et $\mathrm{al}^{16} \mathrm{de}$ muestra una elevación de la proteína $\mathrm{C}$ reactiva y del $\mathrm{FvW}$ en pacientes con CI; estos autores interpretan esta mayor actividad del FvW, considerándola una molécula inflamatoria con valor predictivo de la presencia de CI, en pacientes con factores de riesgo cardiovascular. Este estudio apoyaría más nuestros re- sultados al asociar una elevación de FvW a una mayor expresividad de la CI, independientemente de su valor como reactante de fase aguda, valor demostrado para los niveles de fibrinógeno.

Los pacientes con síntomas de angina a los tres meses de la coronariografía no tuvieron niveles significativamente más altos de FvW que los pacientes asintomáticos, ni hubo diferencias entre los pacientes con ergometría positiva y aquellos con resultado negativo. Esta ausencia de asociación entre los niveles de FvW y el grado de expresividad clínica podría indicar que los niveles del FvW tan solo indicarían una expresión de un riesgo cardiovascular incrementan$\mathrm{do}^{9,30,31}$, aunque en contra de esta interpretación tengamos la relación existente, en el análisis multivariable, entre la presencia de recurrencia clínica y la presencia de más de una lesión coronaria significativa inicialmente.

Los niveles de FvW fueron más elevados a las 24 horas de la coronariografía que antes de dicha prueba. Este resultado podría indicar la expresión de una agresión endotelial inducida por el propio intervencionismo. En este sentido, algunos estudios indican la existencia de la citada agresión endotelial ${ }^{32-37}$, y hay autores que reportan una elevación en los niveles de FvW tras la realización de intervencionismo coronario ${ }^{37}$.

Una de las limitaciones de nuestro estudio es el número limitado de pacientes incluidos en la serie, con unas características heterogéneas, que desemboca en cierto grado de atomización de los resultados. Además, y en relación con la selección del grupo de sujetos sanos, es necesario recordar que la ausencia de enfermedad coronaria es solamente teórica, puesto que obviamente no se realizó coronariografía a los mismos.

Otro inconveniente del estudio sería la producción de un sesgo de observación, puesto que no es posible

TABLA 7. Factor de von Willebrand y situación funcional a los tres meses según la Canadian Cardiovascular Society

\begin{tabular}{|l|c|c|c|c|c|}
\hline & Categoría IV $(\mathrm{n}=1)$ & Categoría III $(\mathrm{n}=8)$ & Categoría II $(\mathrm{n}=14)$ & Categoría I $(\mathrm{n}=12)$ & Asintomáticos $(\mathrm{n}=40)$ \\
\hline $\begin{array}{l}\text { FvW } \\
\text { Test Mood }\end{array}$ & $134 \%$ & $169 \pm 75 \%$ & $131 \pm 47 \%$ & $130,6 \pm 58 \%$ & $125 \pm 63 \%$ \\
\end{tabular}

FvW: factor de von Willebrand. 
descartar que parte de la elevación del FvW se deba a la existencia de múltiples factores de riesgo, factores que per se pueden ser capaces de promover una lesión endotelial ${ }^{19-22,38-40}$. Como ejemplo de este posible sesgo, observamos cómo nuestros resultados demuestran tanto unos menores niveles de c-HDL como unos niveles de colesterol total más elevados en los pacientes que en el grupo control, lo que dificulta la interpretación de nuestro trabajo, al poder haber influido los niveles de lípidos en los niveles de FvW. Por el contrario, la existencia de lesiones coronarias demostradas mediante la angiografía nos permite afirmar que la especificidad de nuestros resultados puede ser elevada, puesto que en el $100 \%$ de los casos se apreció lesión endotelial coronaria, con lo que también podemos deducir que el camino final común de los factores de riesgo cardiovascular desembocaría precisamente en la promoción de una lesión endotelial que conlleva un incremento en el $\mathrm{FvW}^{4-12}$.

\section{Declaración de conflicto de intereses}

Los autores han declarado no tener ningún conflicto de intereses.

\section{BIBLIOGRAFÍA}

1. Jang IK, Lassila R, Fuster V. Atherogenesis and inflammation. Eur Heart J. 1993;14 Suppl K:2-6.

2. Fuster V, Badimon L, Badimon JJ, Chesebro JH. The pathogenesis of coronary artery disease and the acute coronary syndromes (1). N Engl J Med. 1992;326:242-50.

3. Fuster V, Badimon L, Badimon JJ, Chesebro JH. The pathogenesis of coronary artery disease and the acute coronary syndromes (2). N Engl J Med. 1992;326:310-8.

4. Badimon L, Badimon JJ, Turitto VT, Vallabhajosula S, Fuster V. Platelet thrombus formation on collagen type I: a model of deep vessel injury: influence of blood rheology, von Willebrand factor, and blood coagulation. Circulation. 1988;78:1431-42.

5. Ruggeri ZM, Ware J. The structure and function of von Willebrand Factor. Thromb Haemost. 1992;67:594-9.

6. Rand JH, Glanville R, Wu X, Ross JM, Zangari M, Gordon $\mathrm{RE}$, et al. The significance of subendothelial von Willebrand Factor. Thromb Haemostas. 1997; 78:445-50.

7. Boneu B, Abbal M, Plante J, Bierme R. Factor VIII complex and endothelial damage. Lancet. 1975;1:1430.

8. Badimon L, Badimon JJ, Chesebro JH, Fuster V. Von Willebrand factor and cardiovascular disease. Thromb Haemostas. 1993;70:111-8

9. Chung NA, Lydakis C, Belgore F, Li-Saw-Hee FL, Blann AD, Lip GY. Angiogenesis, thrombogenesis, endothelial dysfunction and angiographic severity of coronary artery disease. Heart. 2003;89:1411-5.

10. Morange PE, Simon C, Alessi MC, Luc G, Arveiler D, Ferrieres J, et al. Endothelial cell markers and the risk of coronary heart disease: the Prospective Epidemiological Study of Myocardial Infarction (PRIME) study. Circulation. 2004;109: 1343-8.

11. Makin AJ, Blann AD, Chung NA, Silverman SH, Lip GY. Assessment of endothelial damage in atherosclerotic vascular disease by quantification of circulating endothelial cells. Relationship with von Willebrand factor and tissue factor. Eur Heart J. 2004;25:371-6.

12. Yarnell J, McCrum E, Rumley A, Patterson C, Salomaa V, Lowe G, et al. Association of European population levels of thrombotic and inflammatory factors with risk of coronary heart disease: the MONICA Optional Haemostasis Study. Eur Heart J. 2005;26:332-42.

13. Hoshiba Y, Hatakeyama K, Tanabe T, Asada Y, Goto S. Co-localization of von Willebrand factor with platelet thrombi, tissue factor and platelets with fibrin, and consistent presence of inflammatory cells in coronary thrombi obtained by an aspiration device from patients with acute myocardial infarction. J Thromb Haemost. 2006;4:114-20.

14. Yamashita A, Sumi T, Goto S, Hoshiba Y, Nishihira K, Kawamoto R, et al. Detection of von Willebrand factor and tissue factor in platelets-fibrin rich coronary thrombi in acute myocardial infarction. Am J Cardiol. 2006;97:26-8.

15. Batker HE, Ingerslev J. Plasma concentrations of von Willebrand factor in patients with angina pectoris secondary to coronary atherosclerosis or cardiac syndrome X. Thromb Res. 2000;97:519-23.

16. Danesh J, Wheeler JG, Hirschfield GM, Eda S, Eiriksdottir G, Rumley A, et al. C-reactive protein and other circulating markers of inflammation in the prediction of coronary heart disease. $\mathrm{N}$ Engl J Med. 2004;350:1387-97.

17. Braunwald E. Unstable angina: A classification. Circulation. 1989;80:410-4.

18. Goldman L, Hashimoto B, Cook EF, Loscalzo A. Comparative reproducibility and validity of systems for assessing cardiovascular functional class: advantages of a new specific activity scale. Circulation. 1981;64:1227-34.

19. Folsom AR, Wu KK, Rosamond WD, Sharrett AR, Chambless LE. Prospective study of hemostatic factors and incidence of coronary heart disease: The Atheroesclerosis Risk in Communities (ARIC) study. Circulation. 1997;96:1102-8.

20. Caballero AE, Arora S, Saouaf R. Microvascular and macrovascular reactivity is reduced in subjects at risk for type II diabetes. Diabetes. 1999;48:1856-62.

21. Van Mourik JA, Boertjes R, Huisveld IA, Fijnvandraat K, Pajkrt D, Van Genderen PJ, et al. Von Willebrand factor propeptide in vascular disorders: A tool to distinguish between acute and chronic endothelial cell perturbation. Blood. 1999;94:179-85.

22. Virgós Señor B, Nebra Puertas AC, Suárez Pinilla MA, Cornudella Lacasa R, Portero Pérez P, Morón Giménez A, et al. Elevación del factor de Von Willebrand y D-Dímeros como marcadores de lesión endotelial, en pacientes con hipertensión arterial y cardiopatía isquémica. Hipertensión. 2004;21:290-5.

23. Poletti LF, Bird KE, Marqués D, Harris RB, Suda Y, Sobel M. Structural aspects of heparin responsible for interactions with von Willebrand Factor. Arterioescler Thromb Vasc Biol. 1997; 17: 925-31.

24. Rastegar-Lari G, Legendre P, Ajzenberg N, Warszawaski J, Meyer D, Baruch D. Von Willebrand Factor binding to heparin in various types of von Willebrand disease. Hematol J. 2000;1:190-8.

25. Pernerstorfer T, Speiser W, Stohlawetz P, Eichler H-G, Jilma B. Effects of heparin and aspirin on circulating P-selectin, Eselectin and von Willebrand Factor levels in healthy men. Atherosclerosis. 2001;155:389-93.

26. Montalescot G, Collet P, Lison L, Choussat R, Ankri A, Vicaut E, et al. Effects of various anticoagulant treatments on von Willebrand factor release in unstable angina. J Am Coll Cardiol. 2000;36:110-4.

27. Ray KK, Morrow DA, Gibson CM, Murphy S, Antman EM, Braunwald E. Predictors of the rise in vWF after ST elevation myocardial infarction: implications for treatment strategies and clinical outcome: An ENTIRE-TIMI 23 substudy. Eur Heart J. 2005;26:440-6.

28. Mills J, Mansfield M, Grant P. Elevated fibrinogen in the healthy male relatives of patients with severe, premature coronary artery disease Eur Heart J. 2002;23:1276-81.

29. Montalescot G, Collet JP, Choussat R, Thomas D. Fibrinogen as a risk factor for coronary heart disease. Eur Heart J. 1998; Supl H:H11-7.

30. Lee KW, Lip GY, Tayebjee M, Foster W, Blann M. Circulating endothelial cells, von Willebrand factor, interleukin-6, and prognosis in patients with acute coronary syndromes. Blood. 2005;2:526-32.

31. Whincup PH, Danesh J, Walker M, Lennon L, Thomson A, Appleby $\mathrm{P}$, et al. Von Willebrand factor and coronary heart disea- 
se: prospective study and meta-analysis. Eur Heart J. 2002;23: 1764-70.

32. Harnek J, Zoucas E, Carlemalm E, Cwikiel W. Differences in endothelial injury after balloon angioplasty, insertion of balloonexpanded stents or release of self-expanding stents: An electron microscopic experimental study. Cardiovasc Intervent Radiol. 1999;22:56-61.

33. Pollman MJ, Hall JL, Gibbons GH. Determinants of vascular smooth muscle cell apoptosis after balloon angioplast injury. Influence of redox state and cell phenotype. Circ Res. 2002;84: 113-21.

34. Okura H, Shimodozono S, Hayase M, Bonneau HN, Yock PG, Fitzgerald P. Impact of deep vessel wall injury and vessel stretching on subsequent arterial remodeling after balloon angioplasty: a serial intravascular ultrasound study. Am Heart J. 2002; 144:323-8.

35. Bienvenu JG, Tanguay JF, Chauvet $P$, Merhi Y. Relationship between platelets and neutrophil adhesion and neointimal growth after repeated arterial wall injury induced by angioplasty in pigs. J Vasc Res. 2000;38:153-62.
36. Marmur JD, Merlini PA, Sharma SK, Khaghan N, Torre $\mathrm{SR}$, Israel DH, et al. Thrombin generation in human coronary arteries after percutaneous transluminal balloon angioplasty. J Am Coll Cardiol. 1994;24:1484-91.

37. Virgós Señor B, Nebra Puertas AC, Suárez Pinilla MA. Niveles de FvW e intervencionismo coronario. Med Intensiva. 2005;29 Supl 1:42.

38. Seligman BG, Biolo A, Polanczyk CA, Gross JL, Clausell N. Increased plasma levels of endothelin 1 and von Willebrand factor in patients with type 2 diabetes and dyslipidemia. Diabetes Care. 2000;23:1395-400.

39. Spencer CG, Gurney D, Blann AD, Beevers DG, Lip GY. Von Willebrand factor, soluble P-selectin, and target organ damage in hypertension: a substudy of the Anglo-Scandinavian Cardiac Outcomes Trial (ASCOT). Hypertension. 2002;40:61-6.

40. O'Callaghan PA, Fitzgerald A, Fogarty J, Gaffney P, Hanbidge M, Boran G, et al. New and old cardiovascular risk factors: C-reactive protein, homocysteine, cysteine and von Willebrand factor increase risk, especially in smokers. Eur J Cardiovasc Prev Rehabil. 2005;12:542-7. 